\title{
DSC examination of cartilage damage of patients undergoing shoulder replacement
}

\author{
András Bata ${ }^{1}$ - László G. Nöt ${ }^{1,2} \cdot$ Hajnalka Szabó ${ }^{3} \cdot$ János Cifra $^{4}$ - Dénes Lőrinczy ${ }^{5}$
}

Received: 5 July 2020 / Accepted: 18 November 2020 / Published online: 17 December 2020

(c) The Author(s) 2020

\begin{abstract}
Shoulder replacement is a viable surgical treatment for comminuted proximal humerus fractures and shoulder osteoarthritis. Proper evaluation of hyaline cartilage is essential for the right choice of prosthesis; however, there are only a limited number of studies available investigating the cartilage of glenohumeral joint of patients undergoing shoulder replacement. We hypothesized that differential scanning calorimetry (DSC) could determine the grade of cartilage damage in patients undergoing reversed shoulder replacement, providing insights into the structural changes of the human cartilage samples. Sample of hyaline cartilage of glenohumeral joint was harvested during reversed shoulder replacement of orthopaedic and trauma patients. Thermal parameters were measured using DSC, and radiological examination of glenohumeral joint was also done prior to surgery. We have found that changes in thermal characteristics and DSC scans clearly indicate the severity of osteoarthritis and cartilage damage. The denaturation temperature range and the half-width of the heat flow curves were significantly wider in the orthopaedic samples compared to the control and traumatic ones. The calorimetric enthalpy $\left(\Delta H_{\text {cal }}\right)$ exhibited significant differences in both pathologic samples, compared to the control. The melting temperatures $\left(T_{\mathrm{m}}\right)$ show that structural change caused by orthopaedic condition is greater than the effect of traumatic. Therefore, differential scanning calorimetry could help to determine the grade of cartilage damage in orthopaedic and trauma patients undergoing reversed shoulder replacement.
\end{abstract}

Keywords DSC $\cdot$ Cartilage $\cdot$ Shoulder $\cdot$ Osteoarthritis $\cdot$ Fracture $\cdot$ Reversed shoulder replacement

\section{Introduction}

Shoulder replacement is a viable surgical treatment for comminuted proximal humerus fractures and shoulder osteoarthritis [1-3]. Generally, severe glenohumeral osteoarthritis requires total shoulder replacement and hemiarthroplasties

Dénes Lőrinczy

denes.lorinczy@aok.pte.hu

1 Department of Traumatology and Orthopaedics, Balassa János Teaching Hospital of Tolna County, 5-7 Béri Balogh Ádám street, Szekszárd 7100, Hungary

2 Faculty of Health Sciences, Institute of Physiotherapy and Sport Science, University of Pécs, 4 Vörösmarty street, Pécs 7621, Hungary

3 Department of Radiology, Balassa János Teaching Hospital of Tolna County, 5-7 Béri Balogh Ádám street, Szekszárd 7100, Hungary

4 Department of Pathology, Balassa János Teaching Hospital of Tolna County, 5-7 Béri Balogh Ádám street, Szekszárd 7100, Hungary

5 Department of Biophysics, Medical School, University of Pécs, 12 Szigeti street, Pécs 7624, Hungary are used only in selected cases. Meanwhile, in the case of rotator insufficiencies and rotator cuff tear arthropathies, reversed shoulder replacement is the primary choice [4]. In the case of comminuted 3- and 4-part proximal humerus fractures, hemiarthroplasty could be an applicable alternative to osteosynthesis [5]; however, reversed shoulder arthroplasty should be preferred in elderly patients or with preexisting rotator cuff damages and arthropathies [6].

Details about the condition of cartilage, osteoporosis, inflammation and rotator cuff damage could help to select the right type of implant [7-9]. Accordingly, Toma T. et al. investigated the histopathological changes of humerus head in cuff tear arthropathy (CTA) compared 
to osteoarthritis $(\mathrm{OA})$ and found that the cartilage layer in CTA was thicker, compared to OA [10].

DSC has been found a relevant method in the examination of joint and cartilage diseases [11-13]. For instance, Naumov et al. utilized DSC in the investigation of hyaline cartilage of the fractured human femoral head [14]. DSC has also been successfully used in the examination of degenerative shoulder conditions [15] and spine diseases [16]. Furthermore, Patczai et al. demonstrated that duration of cryopreservation could interfere with the morphology of human cartilage samples [17].

While the knowledge of the condition of the joint surface is crucial for determination of the right type of prosthesis, there are only a limited number of studies available in the literature, investigating the cartilage of glenohumeral joint of patients undergoing shoulder replacement.

Therefore, we hypothesized that DSC could determine the grade of cartilage damage in orthopaedic and trauma patients undergoing reversed shoulder replacement. We also hypothesized that DSC examination could provide further details about the structural changes of the examined cartilage samples.

\section{Materials and methods}

\section{Sample collection}

The $5 \times 5 \times 2.5 \mathrm{~mm}$ sized sample of the hyaline cartilage of the removed humerus head from the estimated glenohumeral contact area (GCA) was harvested during reversed shoulder replacement [18], Additionally, a cylindrical (5 $\mathrm{mm}$ in diameter) part of the glenoid was collected during preparation of the glenoidal component of the prosthesis. Then, samples were properly stored for further measurements, as described in details elsewhere [11, 13]. The hyaline cartilage samples - three from different medical stages-were obtained patients who underwent reversed shoulder replacement: 1) due to acute trauma (4-part dislocated or Neer Type VI proximal humerus fractures (samples A2-4) and 2) due to rotator cuff tear arthropathy (samples B1 and B2). The control sample (originally labelled as A1) was collected from a young patient with dislocated 4-part proximal humerus fracture and was considered macroscopically as a healthy cartilage. This finding was confirmed by histological examination. All of these samples were considered as waste from the point of surgery.

All procedures followed were in accordance with the ethical standards of the responsible Regional and Institutional Research Ethics Committee and with the Helsinki Declaration of 1975, as revised in 2008 .

\section{Clinical and radiological evaluation of rotator cuff damage}

Based on patient history and results of the physical examination on case of rotator cuff arthropathy (RCA) patients, an individual score system was applied to assess the potential level of rotator cuff and cartilage damage. Samples of RCA of trauma origin were also macroscopically assessed, looking for signs of rotator cuff injury and cartilage injury (grade 0-5). The rotator cuff tendon damage was scored with Hamada classification [19]; for evaluation of primary arthropathies and cartilage damage, Walch [20] and Outerbridge classifications [21] were used, respectively. Radiological analysis of conventional X-rays, CT and MRI images was performed by using Siemens Syngo.via software (Siemens Healthineers Global).

\section{Histological processing}

The cartilage samples were fixed in $4 \%$ formaldehyde for at least 2 days. After fixation, two representative sections from each sample were cut. Then, the tissues were dehydrated and embedded in paraffin according to the standard histopathological processing and $4-\mu \mathrm{m}$-thick sections were cut by microtome. The slides were stained with hematoxylin-eosin.

\section{DSC investigations}

Before the measurements, the stored samples were washed three times in normal saline and remained in a sterile isotonic saline on $4{ }^{\circ} \mathrm{C}$ till starting the calorimetric examinations (max. half an hour). The measurements were made by a SETARAM Micro DSC-II calorimeter between 0 and $100{ }^{\circ} \mathrm{C}$ with a heating rate of $0.3 \mathrm{~K} \mathrm{~min}^{-1}$. Conventional closed Hastelloy batch vessels $(V=1 \mathrm{~mL})$ were used for the experiment to perform the thermal denaturation. Samples' masses were in the case of healthy control between in mgs: 157-172. In the case of orthopaedic samples the masses varied in group B1: $55-72$, for B2: $80-90 \mathrm{mg}$. In the case of traumatic samples, it was in the range of 56-66 for A2, 8-11 for A3 and 40-60 mg for A4 patients. Normal saline was used as a reference. With the help of a two-point setting, SETARAM peak integration calorimetric enthalpy $\left(\Delta H_{\text {cal }}\right)$ was calculated from the area under the heat absorption curve and then the other thermal parameters (denaturation or melting temperature $\left(T_{\mathrm{m}}\right)$, range of denaturation $(\Delta T)$, half width of transition $\left(T_{1 / 2}\right)$ and calorimetric enthalpy data of samples) were compared. Because of the relatively small sample mass, the raw scans were smoothed and baseline correction was made by Origin 6.0 . 


\section{Data evaluation}

The average of three measurements for all case was calculated, and the average curves were plotted. Because of the small number of samples, standard deviation was not calculated.

\section{Results}

\section{Radiological and histological evaluation}

Radiological evaluation of rotator cuff tendon damage and glenohumeral arthritis was performed based on the available conventional X-rays, CT and MRI scans (Figs. 1a-d, $2 \mathrm{a}-\mathrm{c})$. Table 1 shows the results of radiological assessment of orthopaedic and trauma shoulders (4-part or Neer Type VI dislocated proximal humerus fractures).

According to the Walch classification, grade ' $\mathrm{D}$ ' in the case of sample B2 indicates a very severe arthritis with anterior humeral head subluxation and level 'A1' in the case of sample A3 means a well-centred humeral head with only minor erosions. Based on MRI scans, Outerbridge 'grade 2' of sample B1 means blister-like swelling/fraying of articular cartilage extending to surface, a moderately damaged cartilage. The Hamada classification indicates the progression of rotator cuff tear arthropathies (from 1 to 5). Of the trauma samples, A3 was graded as ' $4 \mathrm{~b}$ ' and orthopaedic samples B1
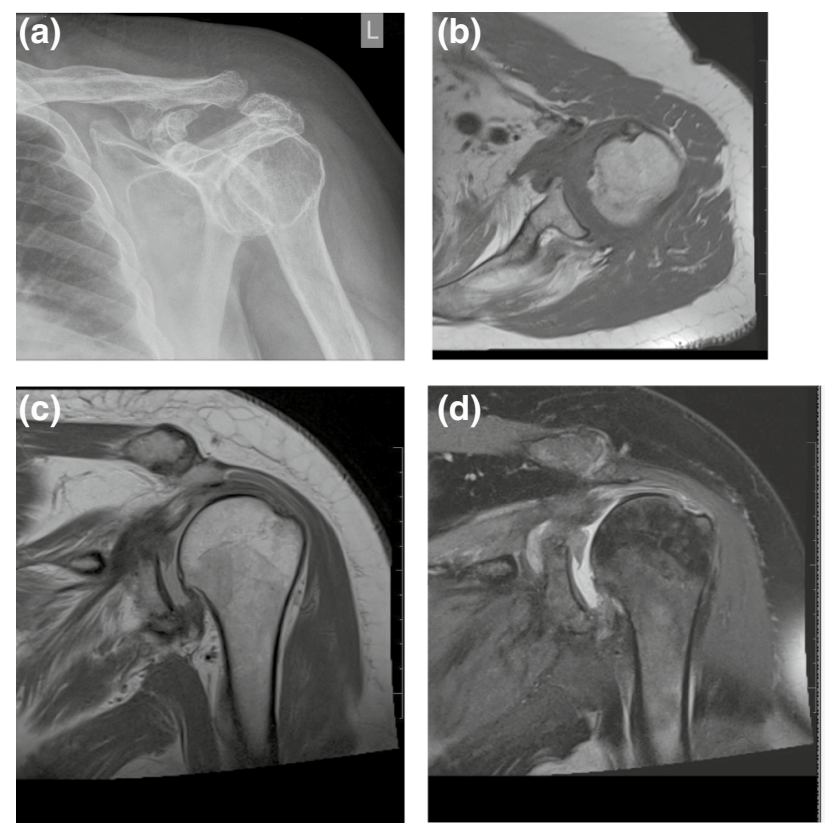

Fig. 1 Rotator cuff tear arthropathy. (a) Conventional X-ray, (b) MRI-T1-weighted, axial plane (c) MRI-PD (proton density) fat saturated, axial plane (c) MRI-PD (proton density), coronal plane, (d) MRI-MRI-PD (proton density), fat saturation, coronal plane
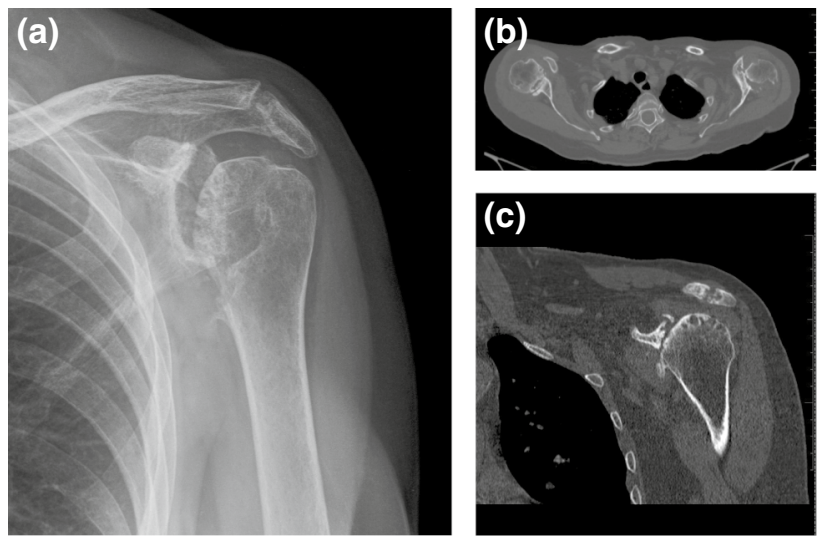

Fig. 2 Rheumatoid arthritis. (a) Conventional X-ray, (b) CT-scan, axial plane, (c) CT-scan, coronal plane

and B2 were graded as ' $4 \mathrm{~A}$ ' and ' 5 ', respectively, demonstrating a worse prognosis of arthropathy for the orthopaedic patients.

The macroscopic findings were scored based on the observation of the surgeon during samples collection. We have found that in the case of the orthopaedic samples, the cartilage was in markedly worse condition, compared to the trauma samples.

It is important to note that most of the classifications found in the literature are applied only for degenerative conditions, not for trauma cases. In the case of healthy, control hyaline cartilage samples, histological examination confirmed the absence of pathologic findings (Fig. 3a).

\section{Thermal denaturation of orthopaedic and trauma samples}

The average curves for orthopaedic and trauma samples are shown in Fig. 4 and in Fig. 5, respectively. The shape and run of scans, as well as the thermal data of Table 2 can

Table 1 Evaluation of glenohumeral arthritis and rotator cuff damage of trauma and orthopaedic shoulders, based on radiological and macroscopic findings

\begin{tabular}{lllll}
\hline \multicolumn{5}{c}{ Classification } \\
\cline { 2 - 5 } & Walch & Outerbridge & Hamada & Macroscopic \\
\hline Orthopaedic & & & $4 \mathrm{a}$ & 4 \\
B1 & - & 2 & 5 & 5 \\
B2 & D & - & & \\
Trauma & & & - & 3 \\
A2 & - & - & $4 \mathrm{~b}$ & 2 \\
A3 & A1 & - & - & $2-3$ \\
A4 & - & - & &
\end{tabular}




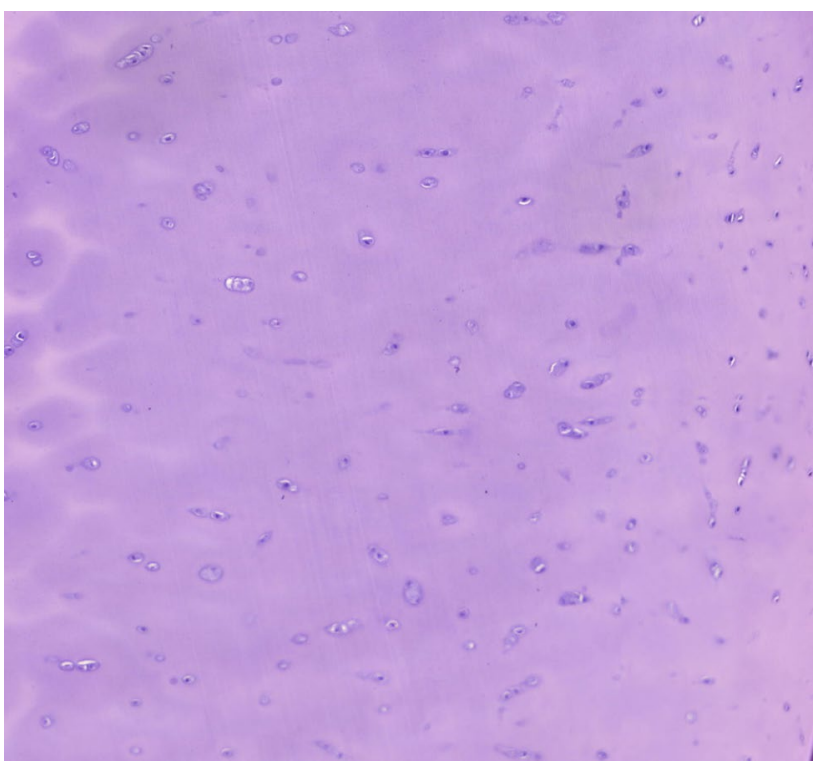

Fig. 3 Histological section from healthy hyaline cartilage. Hematoxylin-eosin staining, magnification is $10 \mathrm{X}$

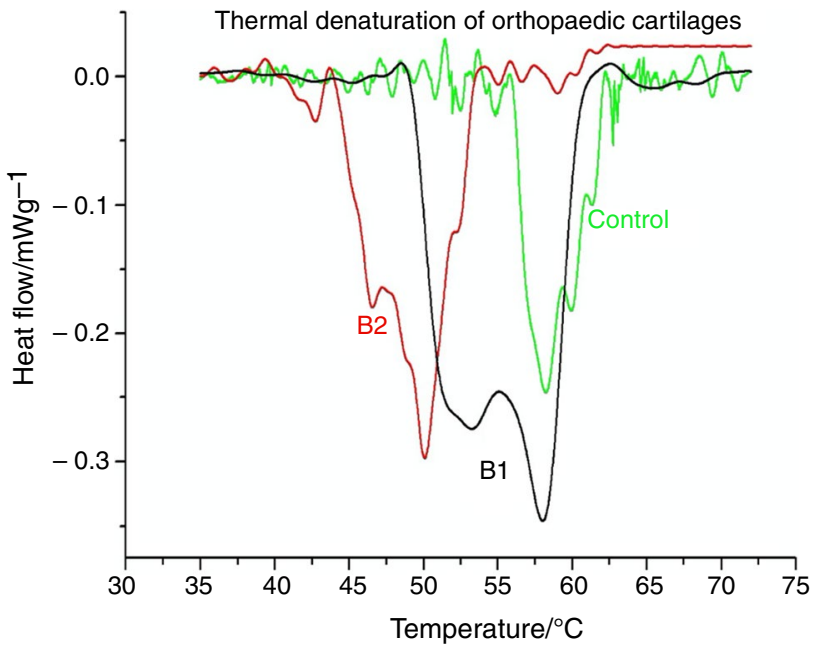

Fig. 4 The average scans of control, B1 and B2 orthopaedic samples of different stages of hyaline cartilage damage

prove the structural changes caused by the actual orthopaedic stage.

Our main observation regarding the origin of the samples and the consequence of the disease is that the denaturation temperature range and the half-width of the heat flow curves are significantly wider in the orthopaedic samples compared to the control and traumatic samples (see Figs. 4, 5 and Table 2). These facts show the thermal consequence of the less cooperativity among the different structural domains of the orthopaedic cartilage.

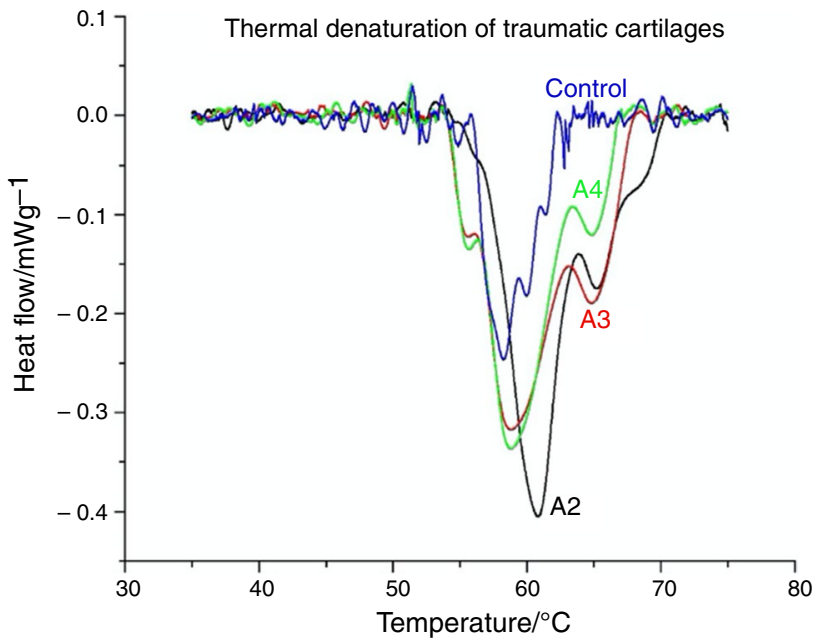

Fig. 5 Thermal denaturation of different traumatic cartilages. The average scans of control, A2-A4 samples of trauma patients with different stages of hyaline cartilage damage

The calorimetric enthalpy exhibited also significant differences in both samples referring to the control. Because we have no biochemical background to separate the different compounds of cartilage, it would be difficult to interpret the 'big jumps' in B1 and B2, compared to the control. The melting temperatures $\left(T_{\mathrm{m}}\right)$ show that structural change caused by disease (B2) is greater than in the case of a traumatic impact.

\section{Discussion}

Despite recently published protocols and guidelines [22-25], there is still a need for studies to analyze the biochemical background of rotator cuff degeneration and hyaline cartilage damage. These data could provide more details to the surgeon about the expected grade of arthritis, especially under circumstances when MRI examination is not available.

In this study, we have demonstrated that changes in the thermal parameters of human cartilage samples could determine the grade of osteoarthritis. We have also found clear differences, comparing DSC scans of trauma and orthopaedic patients.

Based on the DSC curve shapes and thermal characteristics of samples with different origins, we have found that the structural changes caused by degenerative diseases (osteoarthritis, rheumatoid arthritis or rotator cuff tear arthropathy) are greater than changes observed in trauma patients. The structural damage detected in trauma patients could be either due to a preexisting condition or the effect of the trauma itself.

It is known that the human hyaline cartilage consists of three zones (superficial, transitional and deep zone); 
Table 2 Thermal characteristics of orthopaedic and traumatic cartilage samples. Data are averages of three samples. Symbols: $\Delta T$ denaturation temperature range, $T_{1 / 2}$ half width at the highest heat flow, $T_{\mathrm{m}}$ the melting temperature and $\Delta H_{\text {cal }}$ is the calorimetric enthalpy normalized on the wet mass of the samples

\begin{tabular}{|c|c|c|c|c|}
\hline \multirow[t]{2}{*}{ Sample } & \multicolumn{4}{|c|}{ Thermodynamic parameters } \\
\hline & $\Delta T /{ }^{\circ} \mathrm{C}$ & $T_{1 / 2} /{ }^{\circ} \mathrm{C}$ & $T_{\mathrm{m}}{ }^{\circ} \mathrm{C}$ & $\Delta H_{\mathrm{cal}} / \mathrm{Jg}^{-1}$ \\
\hline \multicolumn{5}{|c|}{ Orthopaedic cartilage } \\
\hline Control & 19.0 & 7.9 & 56.5 & 0.62 \\
\hline B1 & 27.1 & 17.6 & 56.0 & 1.84 \\
\hline B2 & 20.0 & 10.5 & 50.0 & 1.04 \\
\hline \multicolumn{5}{|c|}{ Traumatic cartilage } \\
\hline Control & 19.0 & 7.9 & 56.5 & 0.62 \\
\hline A2 & 17.1 & 4.3 & 61.1 & 0.41 \\
\hline A3 & 15.0 & 8.9 & 58.9 & 1.07 \\
\hline A4 & 14.6 & 5.0 & 58.9 & 1.09 \\
\hline
\end{tabular}

each zone has different function and consistency [26]. Interestingly, we have found that the thermal denaturation scans of certain samples exhibited 'jumps' that can be an interference of one of the components of the different zones. Separation of the different layers of the cartilage is beyond the scope of our current study; however, it might worth further investigations.

In comparison, Naumov et al. investigated the effect of time elapsed from the fracture of femoral neck on thermal parameters. They demonstrated differences in both melting temperature and thermal enthalpy, comparing control, fresh fractures and different stages of avascular necrosis (control, fresh fractures: $T_{\mathrm{m}}=68.2^{\circ} \mathrm{C}, \Delta H_{\text {cal }}=2.87 \mathrm{~J} \mathrm{~g}^{-1}$, avascular necrosis: $T_{\mathrm{m}}=70.7{ }^{\circ} \mathrm{C}, \Delta H_{\text {cal }}=3.61 \mathrm{~J} \mathrm{~g}^{-1}$ ). It supports the notion that human hyaline cartilage suffers significant structural changes in the case of inadequate blood supply, such as avascular femoral head necrosis [14].

Potential limitation of the study is the relatively low number of cases in which radiological scoring was available, based on CT/MRI scans. It highlights the problem that most of the classification either utilizes MRI or evaluates only orthopaedic cases, i.e. RCA or OA shoulders, but not traumas. Meanwhile, the proper assessment of hyaline cartilage is essential in the decision-making process and surgical planning, especially in trauma cases.

Another limitation of this study is that only the average values were calculated instead of standard deviation and error of means (because of the low number of patients). However, considering the excellent reproducibility of the measurements, we believe that DSC produces marked differences, therefore a useful examination method in the assessment of cartilage damage of patients undergoing shoulder replacement.

\section{Conclusions}

Differential scanning calorimetry could help to determine the grade of cartilage damage in orthopaedic and trauma patients undergoing reversed shoulder replacement.

Acknowledgements This work was supported by CO-272 Grant (for D.L., National Scientific Research Fund).

Authors' contributions Dr. András Bata was involved in sample collection and handling, data analysis and manuscript writing. Dr. László G Nöt was involved in operations, sample collection and handling, data analysis and manuscript writing. Dr. János Cifra was involved in histological examination. Dr. Hajnalka Szabó was involved in radiological evaluation. Prof. Dr. Dénes Lórinczy is corresponding author and principal investigator and was involved in DSC experiments, data analysis and manuscript writing.

Funding Open access funding provided by University of Pécs..

Availability of data and material There are no additional available data to upload.

\section{Compliance with ethical standards}

Conflict of interest The authors declare that they have no known competing financial interests or personal relationships that could have appeared to influence the work reported in this paper.

Consent to participate Consent from patients to participate in the study has been obtained.

Consent for publication Copyright form has been uploaded with the manuscript.

Ethical approval All procedures followed were approved in accordance with the ethical standards of the responsible committee on human experimentation (institutional and national) and with the revised Helsinki Declaration of 1975. 
Open Access This article is licensed under a Creative Commons Attribution 4.0 International License, which permits use, sharing, adaptation, distribution and reproduction in any medium or format, as long as you give appropriate credit to the original author(s) and the source, provide a link to the Creative Commons licence, and indicate if changes were made. The images or other third party material in this article are included in the article's Creative Commons licence, unless indicated otherwise in a credit line to the material. If material is not included in the article's Creative Commons licence and your intended use is not permitted by statutory regulation or exceeds the permitted use, you will need to obtain permission directly from the copyright holder. To view a copy of this licence, visit http://creativecommons.org/licenses/by/4.0/.

\section{References}

1. Steinhaus ME, Gowd AK, Hurwit DJ, Lieber AC, Liu JN. Return to work after shoulder arthroplasty: a systematic review and metaanalysis. J Shoulder Elb Surg US. 2019;28:998-1008.

2. Collin P, Hervé A, Walch G, Boileau P, Muniandy M, Chelli M. Mid-term results of reverse shoulder arthroplasty for glenohumeral osteoarthritis with posterior glenoid deficiency and humeral subluxation. J Shoulder Elb Surg US. 2019;28:2023-30.

3. Fitschen-Oestern S, Behrendt P, Martens E, Finn J, Schiegnitz J, Borzikowsky C, et al. Reversed shoulder arthroplasty for the treatment of proximal humerus fracture in the elderly. J Orthop. 2020;17:180-6.

4. Killian ML, Cavinatto L, Galatz LM, Thomopoulos S. Recent advances in shoulder research. Arthritis Res Ther. 2012;14:214.

5. Agarwal S, Rana A, Sharma RK. Functional outcome after primary hemiarthroplasty in three or four part proximal humerus fracture: a short term followup. Indian J Orthop. 2016;50:590-4.

6. Garrigues GE, Johnston PS, Pepe MD, Tucker BS, Ramsey ML, Austin LS. Hemiarthroplasty versus reverse total shoulder arthroplasty for acute proximal humerus fractures in elderly patients. Orthop US. 2012;35:e703-8.

7. Stone MA, Namdari S. Surgical considerations in the treatment of osteoporotic proximal humerus fractures. Orthop Clin North Am US. 2019;50:223-31.

8. Holschen M, Siemes M-K, Witt K-A, Steinbeck J. Five-year outcome after conversion of a hemiarthroplasty when used for the treatment of a proximal humeral fracture to a reverse total shoulder arthroplasty. Bone Joint J Engl. 2018;100-B:761-6.

9. Mata-Fink A, Meinke M, Jones C, Kim B, Bell J-E. Reverse shoulder arthroplasty for treatment of proximal humeral fractures in older adults: a systematic review. J Shoulder Elb Surg US. 2013;22:1737-48.

10. Toma T, Suenaga N, Taniguchi N, Oizumi N, Yamaguchi $\mathrm{H}$, Tome $\mathrm{Y}$, et al. Humeral head histopathological changes in cuff tear arthropathy. J Orthop Surg Hong Kong Engl. 2019;27:2309499018816428.

11. Lórinczy D. Thermal analysis in biological and medical applications. J Therm Anal Calorim. 2017;130:1263-80.

12. Nöt LG, Naumov I, Vámhidy L, Lőrinczy D, Wiegand N. Comparison of thermal characteristics of degenerated and inflamed human collagen structures with differential scanning calorimetry. J Therm Anal Calorim. 2013;113:273-9.

13. Wiegand N, Naumov I, Vámhidy L, Kereskai L, Lôrinczy D, Nöt LG. Comparative calorimetric analysis of 13 different types of human healthy and pathologic collagen tissues. Thermochim Acta. 2013;568:171-4.

14. Naumov I, Wiegand N, Patczai B, Vámhidy L, Lőrinczy D. Differential scanning calorimetric examination of the human hyaline cartilage of the femoral head after femoral neck fracture. J Therm Anal Calorim. 2012;108:59-65.

15. Csotye J, Aigner Z, Sohár G, Szabó-Révész P. Tóth K Calorimetric properties of degenerative human shoulder joint hyaline cartilage. J Therm Anal Calorim. 2009;95:805-8.

16. Mécs L, Aigner Z, Sohár G, Szabó-Révész P, Tóth K. Characterization of human cartilage in degenerated spine disease with differential scanning calorimetry. J Therm Anal Calorim. 2009;95:809-11.

17. Patczai B, Mintál T, Nőt LG, Wiegand N, Lőrinczy D. Effects of deep-freezing and storage time on human femoral cartilage. J Therm Anal Calorim. 2017;127:1177-80.

18. Soslowsky LJ, Flatow EL, Bigliani LU, Pawluk RJ, Ateshian GA, Mow VC. Quantitation of in situ contact areas at the glenohumeral joint: a biomechanical study. J Orthop Res Off Publ Orthop Res Soc US. 1992;10:524-34.

19. Brolin TJ, Updegrove GF, Horneff JG. Classifications in brief: Hamada classification of massive rotator cuff tears. Clin Orthop Relat Res. 2017;475:2819-23.

20. Bercik MJ, Kruse K 2nd, Yalizis M, Gauci M-O, Chaoui J, Walch G. A modification to the Walch classification of the glenoid in primary glenohumeral osteoarthritis using three-dimensional imaging. J Shoulder Elb Surg US. 2016;25:1601-6.

21. Slattery C, Kweon CY. Classifications in brief: outerbridge classification of chondral lesions. Clin Orthop Relat Res. 2018;476:2101-4.

22. Smith GCS, Bateman E, Cass B, Damiani M, Harper W, Jones H, et al. Reverse shoulder arthroplasty for the treatment of proximal humeral fractures in the Elderly (ReShAPE trial): study protocol for a multicentre combined randomised controlled and observational trial. Trials. 2017;18:91.

23. Launonen AP, Fjalestad T, Laitinen MK, Lähdeoja T, Ekholm $\mathrm{C}$, Wagle T, et al. Nordic innovative trials to evaluate osteoporotic fractures (NITEP) collaboration: the Nordic DeltaCon trial protocol-non-operative treatment versus reversed total shoulder arthroplasty in patients 65 years of age and older with a displaced proximal humerus fracture: a prospective, randomised controlled trial. BMJ Open. 2019;9:e024916.

24. Harjula JNE, Paloneva J, Haapakoski J, Kukkonen J, Äärimaa V. Increasing incidence of primary shoulder arthroplasty in Finland-a nationwide registry study. BMC Musculoskelet Disord. 2018;19:245.

25. Wilcox RB, Arslanian LE, Millett P. Rehabilitation following total shoulder arthroplasty. J Orthop Sports Phys Ther US. 2005;35:821-36.

26. Sophia Fox AJ, Bedi A, Rodeo SA. The basic science of articular cartilage: structure, composition, and function. Sports Health SAGE Publ. 2009;1:461-8.

Publisher's Note Springer Nature remains neutral with regard to jurisdictional claims in published maps and institutional affiliations. 